\title{
ARTICLE OPEN Bio-inspired flexible artificial synapses for pain perception and nerve injuries
}

\author{
Qifeng Lu ${ }^{1}$, Fuqin Sun ${ }^{1}$, Lin $\mathrm{Liu}^{2}$, Lianhui $\mathrm{Li}^{1}$, Mingming Hao ${ }^{1}$, Zihao Wang ${ }^{1}$ and Ting Zhang ${ }^{1 凶}$
}

Imitation of the perception system of living creatures is of great importance for the construction of artificial nerves and intelligent human-machine interfaces. However, a prominent challenge is to emulate the functions of the biological synapse, which is the basic building block of the neural system. Here, inspired by the pain perception mechanism of the living creatures, a flexible double-layer memristor was constructed, with $90 \%$ semiconducting single-wall carbon nanotubes (s-SWCNTs) covered by LiClO ${ }_{4}$ doped polyoxyethylene oxide $\left(\mathrm{PEO}: \mathrm{LiClO}_{4}\right)$ as the channel materials. The carriers (protons and $\mathrm{Li}^{+}$) from $\mathrm{PEO}$ : $\mathrm{LiClO}_{4}$ imitated the functions of $\mathrm{Na}^{+}$and $\mathrm{K}^{+}$in biological systems. A potentiation of the post-synaptic signal was observed with mild stimuli, while the post-synaptic signal was inhibited with severe stimuli with a pulse voltage larger than $1.4 \mathrm{~V}$ in this research. These behaviors resemble the sensation of pain, neuroprotection, and possible injuries to the neural system. To explore the underlying mechanism of the phenomenon, the fourier-transform infrared spectroscopy (FTIR), X-ray photoelectron spectroscopy (XPS), Raman spectrum, and current (IV) sweep were carried out. It was inferred that the observed results are attributable to the interaction between carriers in PEO: $\mathrm{LiClO}_{4}$ and functional groups and defects in the s-SWCNTs. The enhanced channel current results from the fulfillment of the traps by the carriers, and the suppression of the current is due to the intercalation of $\mathrm{Li}^{+}$in the s-SWCNTs. This flexible artificial synapse opens a new avenue for the construction of biocompatible electronic devices towards artificial intelligence systems.

npj Flexible Electronics (2020)4:3; https://doi.org/10.1038/s41528-020-0066-0

\section{INTRODUCTION}

Pain, a self-protective response of living creatures, is a symptom that may be caused by a disease or an acute stimulus. The pain signal, activated by nociceptors distributed at the peripheral terminals, is transmitted to the central nervous system via dorsal root ganglion (DRG) or trigeminal ganglion neurons ${ }^{1-3}$. The sensation of pain is an important adaptive mechanism in biological systems. On the one hand, it can protect a person from further injury by transmitting pain-causing signals such as heat, pressure and chemical agents to the somatosensory thalamus. Then, the subsequent protective responses can be made immediately. On the other hand, pathological and severe damage pain can be limited by suppressing the neuronal excitation through the activation of TREK2 channels, which are known to be neuroprotective agents ${ }^{4,5}$. Thus, the overexpression of the neuron signal can be inhibited in cases of neuropathic injury of the nervous system due to painful stimuli. To uncover the pain perception mechanisms, a great amount of effort has been made, and many studies have demonstrated that various ion channels are involved in the development of pain perception ${ }^{6-8}$. Recently, with the development of molecular cell biology and genetic engineering, the molecular basis of ion channels has been elucidated. It is believed that voltage-controlled $\mathrm{Na}^{+}$and $\mathrm{K}^{+}$ channels are involved in the sensation and transmission of pain $^{3,9,10}$. In brief, the membrane potentials of the peripheral nerve terminals are depolarized when the stimuli activate peripheral terminals through nociceptive ion channels. Then, an action potential is induced through the activation of voltagedependent $\mathrm{Na}^{+}$channels once the threshold of the membrane potential is reached. In contrast, the mechanism for suppressing excitability by a negative membrane potential is proved to be related to hyperpolarization of the membrane potential by leak $\mathrm{K}^{+}$ channels. This behavior leads to a decrease in the conduction fidelity across the axon or limited neurotransmitter release at the axon terminals, which prevents the neuron from generating an action potential. Therefore, ion channels are crucial determinants for the adaptive mechanism of pain sensation.

Artificial synapses, which can emulate most of the functions of biological synapses, have attracted a tremendous amount of attention ${ }^{11-13}$. Much effort has been made to study the device structures, material systems, and switching mechanisms to demonstrate synaptic characteristics and improve the device performance since synaptic behaviors were mimicked by Mead in 1996 using a floating-gate silicon MOS transistor ${ }^{14}$. For example, memristor- and transistor-based artificial synapses with coplanar and vertical structures made of metal oxides, organic/inorganic perovskites and two-dimensional materials have been investigated $^{15-20}$. Based on these synaptic devices, a number of biologically inspired functions, such as visual information processing, speech recognition, and movement control, have been realized. In addition, benefiting from the development of biologically inspired systems, great achievements have occurred in many fields, including neuromorphic computing ${ }^{21,22}$, bioinspired sensing systems ${ }^{23}$, control theory for legged robots ${ }^{24,25}$, and prosthetics ${ }^{26,27}$. However, the emulation of pain perception using synaptic devices has not been reported despite progress in the design and fabrication of artificial synapses.

Herein, inspired by the working mechanism of the pain perception system of living creature, a memristor-based structure artificial synapse with double-layer active materials was designed, in which $\mathrm{LiClO}_{4}$ doped polyoxyethylene (PEO: $\mathrm{LiClO}_{4}$ ), a bioadhesive, mucoadhesive, and biocompatible polymer, was

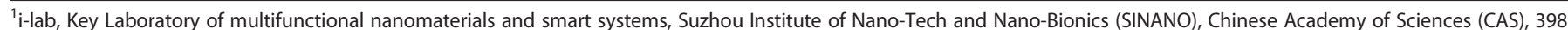

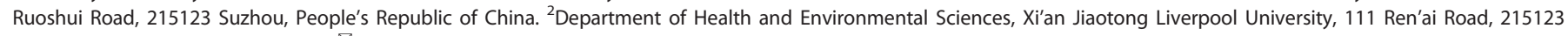
Suzhou, People's Republic of China. ${ }^{凶}$ email: tzhang2009@sinano.ac.cn
} 
(a)

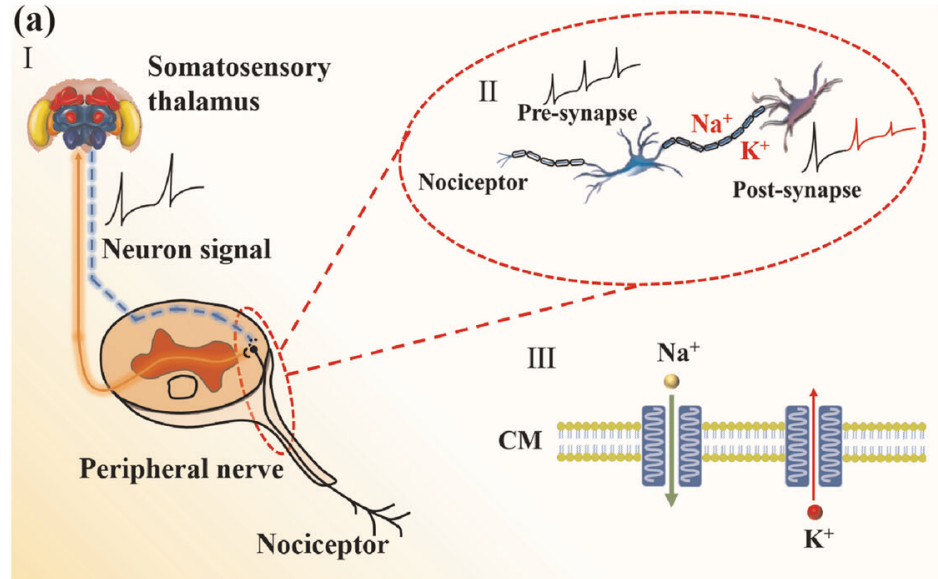

(b)
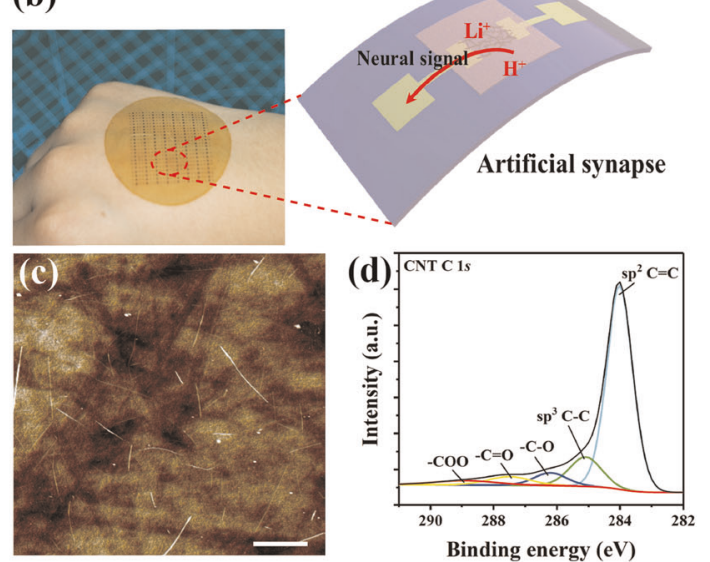
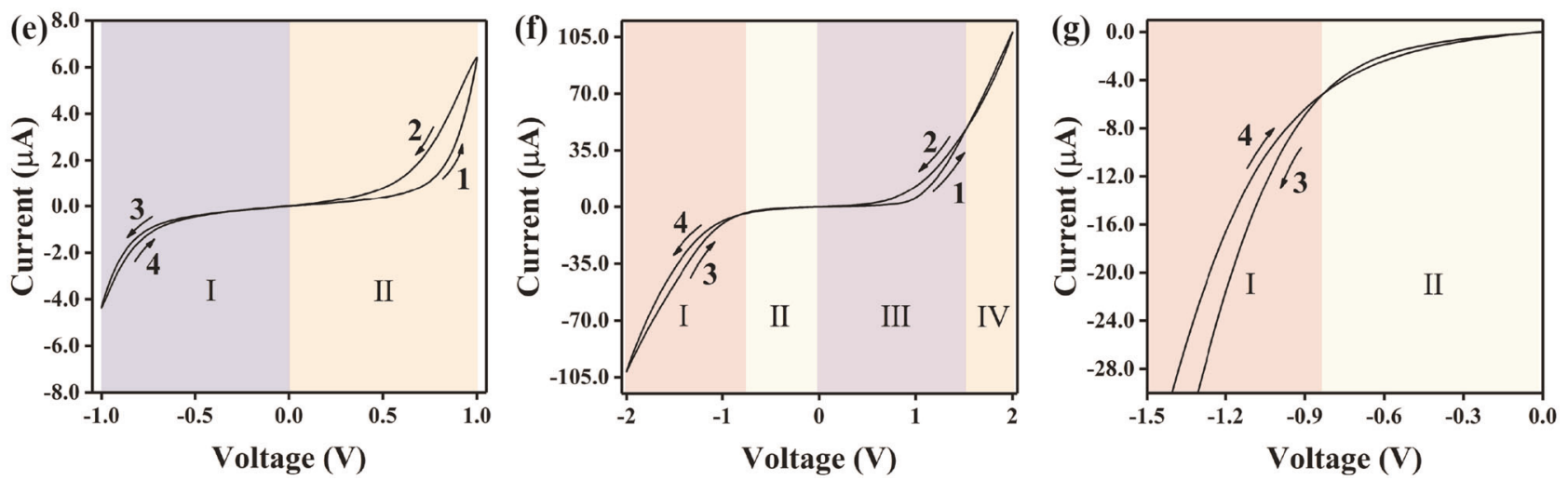

Fig. 1 Design of the bio-inspired double-layer memristor. a The schematic diagram for the pain perception mechanisms of the creature. $\mathbf{b}$ The optical image of the memristor-based artificial synapse, in which s-SWCNTs and PEO: $\mathrm{LiClO}_{4}$ are used as the bottom-layer and top-layer materials, respectively. The protons and $\mathrm{Li}^{+}$mimic the functions of $\mathrm{Na}^{+}$and $\mathrm{K}^{+}$in biological systems. c The AFM image with the length of the scale bar being $1 \mu \mathrm{m}$ and $\mathbf{d}$ the XPS spectra of s-SWCNTs. The IV characteristics of the artificial synapses with the different sweeping voltages shown in $\mathbf{d}-\mathbf{f}$ The enlarged IV characteristics in the voltage between $0 \mathrm{~V}$ and $-1.5 \mathrm{~V}$.

selected as the top-layer material. The protons and $\mathrm{Li}^{+}$in this layer behave like $\mathrm{Na}^{+}$and $\mathrm{K}^{+}$in biological systems. $90 \%$ semiconducting single-wall carbon nanotubes (s-SWCNTs) were used as the bottom-layer material due to their unique physical, electrical, and mechanical properties and easy modulation of the electrical charges in SWCNTs by altering their surrounding electrical environment ${ }^{28}$. The observed channel current is attributed to the hopping of carriers through functional groups. In addition, it can be enhanced due to the reduction in the barrier resulting from the fulfillment of the traps and limited by the intercalation of the $\mathrm{Li}^{+}$in s-SWCNTs. Therefore, $\mathrm{Na}^{+}$and $\mathrm{K}^{+}$dynamics in biological systems can be mimicked by the cooperation of protons and $\mathrm{Li}^{+}$ in artificial synapses. Consequently, the pain perception and possible nerve injuries caused are successfully emulated.

\section{RESULTS}

Figure 1(a-l) shows a schematic diagram of the pain perception mechanisms of living creatures. The pain-causing signals, such as external stimuli and tissue disease, are detected by receptors distributed at the nociceptor peripheral terminals. Then, the painsignal is transmitted to the sensory cortex of the somatosensory thalamus via the afferent nerve (DRG or trigeminal ganglion neurons) ${ }^{3}$. Once the sensation of pain occurs, appropriate protective responses are taken either via a nervous reflex or commands from the brain. In this process, $\mathrm{Na}^{+}$plays an important role in neuronal excitability and neuron signal transmission. In addition, the central nervous system utilizes descending inhibitory pathways to modulate the transmission of nociceptive stimuli, which is considered as a neuroprotective and adaptive mechanism of the neural system ${ }^{2} . \mathrm{K}^{+}$plays an important role in the inhibition of neuronal excitability by counteracting action potential, limiting neurotransmitter release at the axon terminals $^{29}$. This neuroprotective mechanism can suppress the overexpression of the neuron signal in the case of neuropathic injury of the nervous system due to acute painful stimulus stimuli. The roles of $\mathrm{Na}^{+}$and $\mathrm{K}^{+}$are shown in Fig. $1(\mathrm{a}-\mathrm{II})$ and (a-III). Figure $1 \mathrm{~b}$ shows the optical image and schematic diagram of the flexible artificial synapses. In this device, protons and $\mathrm{Li}^{+}$were employed to mimic the functions of $\mathrm{Na}^{+}$and $\mathrm{K}^{+}$in biological synapses because the electrical properties can be modulated easily by the intercalation of $\mathrm{Li}^{+}$into s-SWCNTs, benefiting from its smaller atomic sizes. In addition, no additional impurities need to be introduced if the protons are employed as charge carriers. The atomic force microscope (AFM) image of the s-SWCNTs, the bottom-layer material of the memristor, is shown in Fig. 1c. The XPS spectra in Fig. 1d show the presence of functional groups in the s-SWCNTs, which contribute to the hopping process of the carriers. The detailed fabrication procedure of the artificial synapses is presented in the methods section and Supplementary Figs 1 and 2. A distinguished behavior in the IV characteristics for different sweeping voltages was observed and is shown in Fig. 1e, f. An anticlockwise hysteresis was measured with a small sweeping voltage of $1 \mathrm{~V}$, which indicated a synaptic potentiation. However, with the increase in sweeping voltage, $2 \mathrm{~V}$ in this research, a clockwise hysteresis was observed at the voltages 
(a)

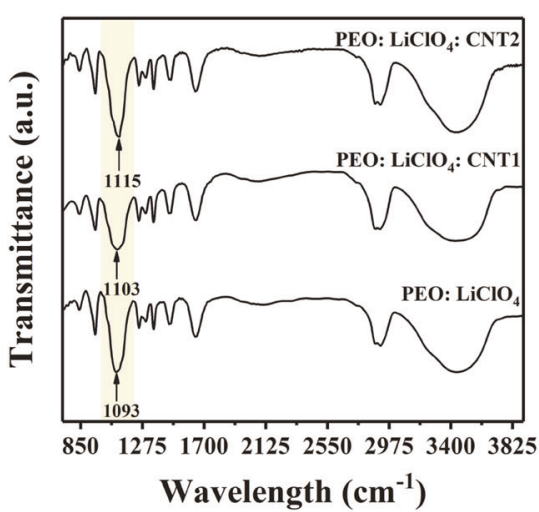

(c)

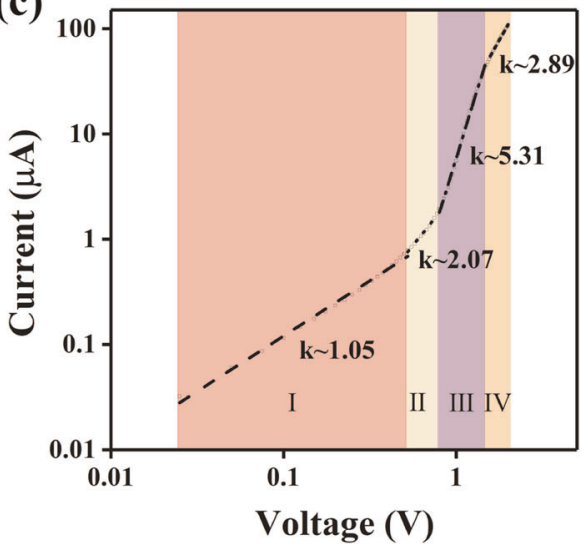

(b)

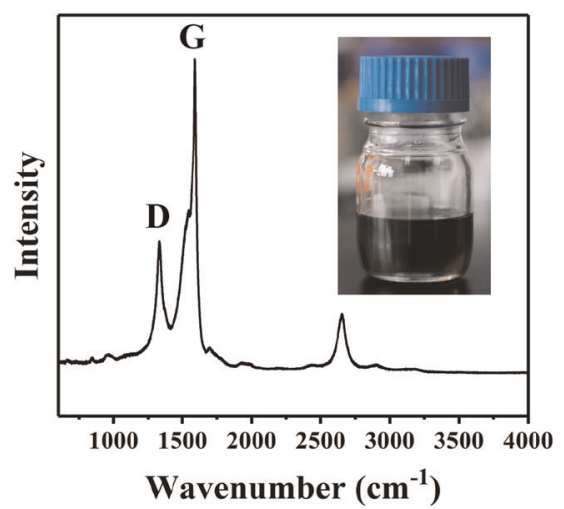

(d)

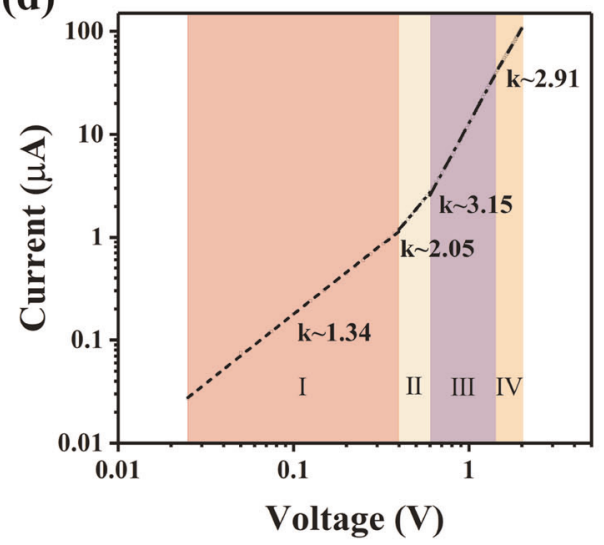

Fig. 2 Investigation of the underlying mechanism for the synaptic behaviors. a The FTIR spectra for PEO: $\mathrm{LiClO}_{4}, \mathrm{PEO}: \mathrm{LiClO}_{4}: \mathrm{CNT1}$, and PEO: $\mathrm{LiClO}_{4}$ : CNT2. Changes in the highlited frequency region indicates the interaction between s-SWCNTs and PEO: LiClO 4 . $\mathbf{b}$ The presence of D-band in Raman spectra is a diagnostic of disorder and sidewall defects. The relative intensity of the D-band to G-band of 0.43 indicating the functionalization of the s-SWCNTs. The ionic behaviors were uncovered by the investigation of the conduction mechanism for the forward and reverse IV characteristics plotted in log scale in $\mathbf{c}$, $\mathbf{d}$.

between $1.4 \mathrm{~V}$ and $2 \mathrm{~V}$, which implies synaptic inhibition. The enlarged IV characteristics in the voltage between $0 \mathrm{~V}$ and $-1.5 \mathrm{~V}$ are shown in Fig. $1 \mathrm{~g}$ to provide a clear picture of the intersection of the clockwise and anticlockwise hysteretic loops. Moreover, almost no degradation of the device performance is observed under the bending condition and even for a small bend radius of $\sim 6 \mathrm{~mm}$ as shown in Fig. 3, which demonstrates the flexibility of the device.

To explore the underlying mechanism for the voltagedependent potentiation and inhibition behaviors of the artificial synapse, the physical and electrical properties of s-SWCNTs and PEO: $\mathrm{LiClO}_{4}$ and their possible interactions were investigated. Supplementary Fig. 4a, b show the typical IV curves for the devices with s-SWCNTs or PEO: $\mathrm{LiClO}_{4}$ as the channel materials. In addition, the current was two orders of magnitude smaller than that measured from the double-layer artificial synapse. These results imply that the observed memristive behaviors described in Fig. If are attributed to the interaction between s-SWCNTs and PEO: $\mathrm{LiClO}_{4}$. From the FTIR spectra in Fig. 2a for PEO: $\mathrm{LiClO}_{4}, \mathrm{PEO}: \mathrm{LiClO}_{4}$ : CNT1, and PEO: $\mathrm{LiClO}_{4}$ : CNT2 (PEO: $\mathrm{LiClO}_{4}$ : CNT2 has a higher sSWCNT concentration than PEO: $\mathrm{LiClO}_{4}$ : CNT1), a frequency region is of particular interest. The band at $1093 \mathrm{~cm}^{-1}$ observed in the FTIR spectrum of PEO: $\mathrm{LiClO}_{4}$ originates from the $\mathrm{C}-\mathrm{O}-\mathrm{C}$ stretch. This confirms the presence of crystalline PEO, which is also supported by the optical images and X-ray diffraction (XRD) patterns in Supplementary Fig. 5. When s-SWCNTs were blended into the PEO: $\mathrm{LiClO}_{4}$ matrix, a redshift of the $\mathrm{C}-\mathrm{O}-\mathrm{C}$ stretch was observed with the peak shifting to $1103 \mathrm{~cm}^{-1}$ and $1115 \mathrm{~cm}^{-1}$, respectively, for low- and high-s-SWCNT doping concentrations. Additionally, the crystallinity of the blended materials decreased with increasing s-SWCNT concentration, as proved by the XRD patterns in Supplementary Fig. $5 \mathrm{~d}$. This phenomenon is due to the wrapping of carbon nanotubes by PEO: $\mathrm{LiClO}_{4}$. The carbon nanotubes with large surface areas and many functional groups indicated by the XPS spectra in Fig. 1d can disrupt the chain folding and restrict the organization of PEO: $\mathrm{LiClO}_{4}$ chains, leading to a decrease in the crystallinity and an enhancement in the ionic conductivity. As a result, the enhanced ionic conductivity contributes to an obvious increase in the current of the artificial synapse compared with those of s-SWCNTs or PEO: $\mathrm{LiClO}_{4}$ as the channel materials independently.

With the known interaction of s-SWCNTs and PEO: $\mathrm{LiClO}_{4}$, the ionic dynamics of the artificial synapses must be investigated. First, anticlockwise and clockwise hysteretic loops were measured in the small $(0-1.4 \mathrm{~V})$ and large (1.4-2 V) voltage sweeping regions, respectively, for the artificial synapse discussed in Fig. 1. However, the IV characteristics extracted from the device with s-SWCNTs covered by chitosan (CS) as the channel material (Supplementary Fig. 6) show an anticlockwise hysteresis regardless of the sweeping voltage. This outcome is believed to be a result of the hopping and trapping of the protons, as reported in previous research ${ }^{30,31}$. From this comparison, it can be inferred that there are two different carriers contributing to the memristive behaviors of the artificial synapse in this research. Since the PEO: $\mathrm{LiClO}_{4}$ membrane was employed as the top-layer material, it is considered that protons and $\mathrm{Li}^{+}$play significant roles in the ionic 


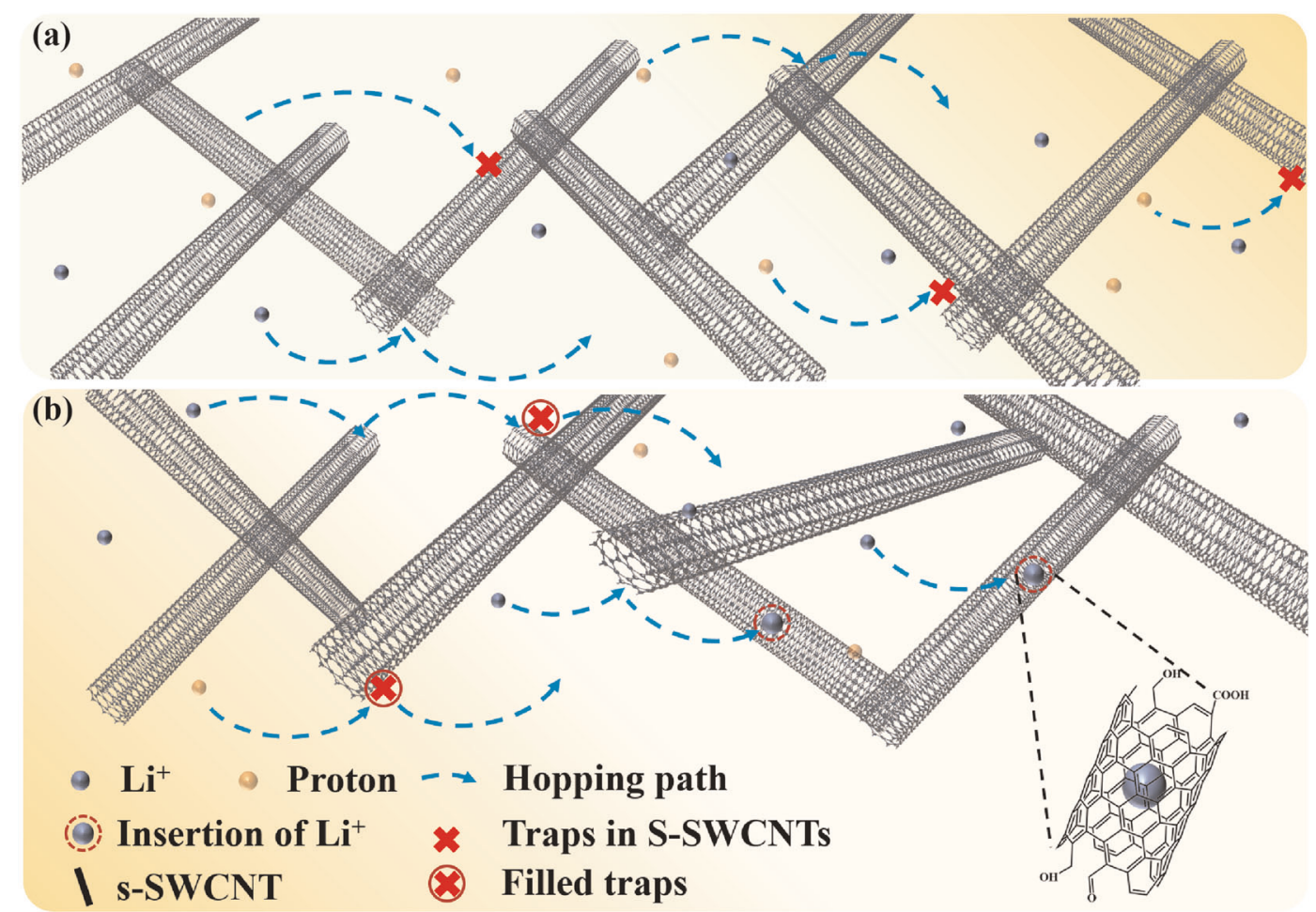

Fig. 3 The schematic diagram for the working mechanism of the artificial synapse. a In low-voltage region, the hopping of carriers through functional groups and their trapping in the trapping-sites through topological defects. $\mathbf{b}$ In high-voltage region, the intercalation of $\mathrm{Li}^{+}$in the s-SWCNTs occurs, which leads to a reduction in carrier concentration and mobility.

(a)

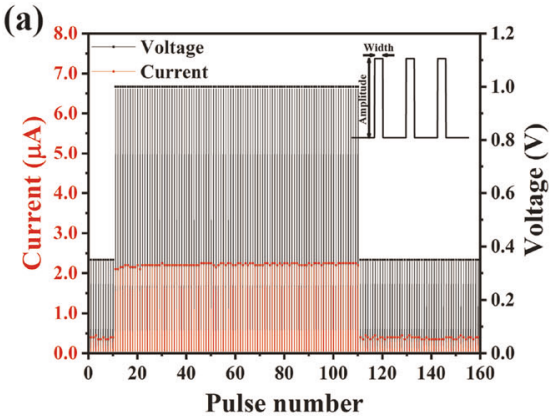

(d)

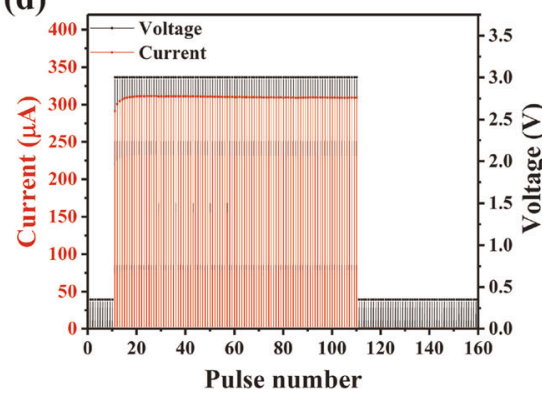

(b)

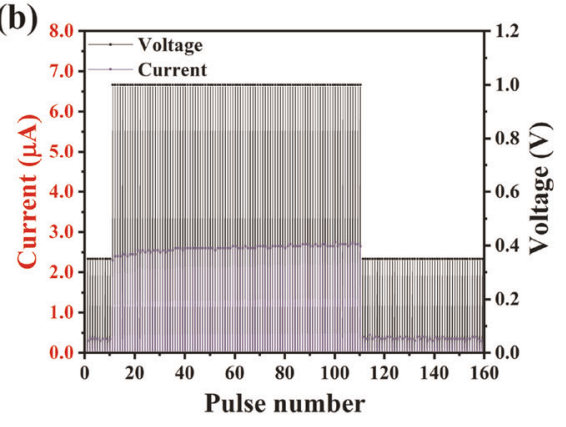

(e)

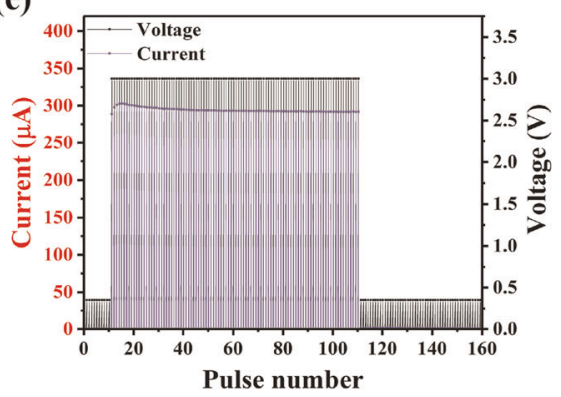

(c)

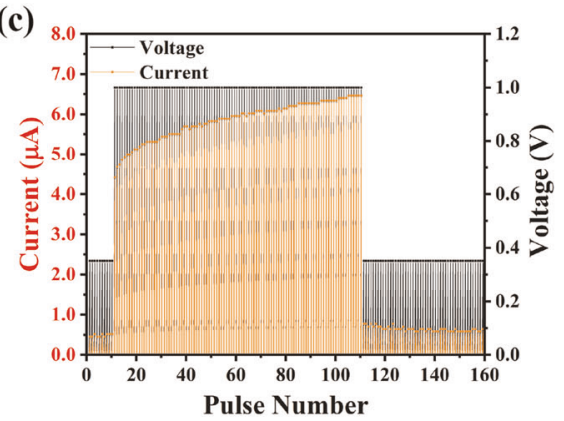

(f)

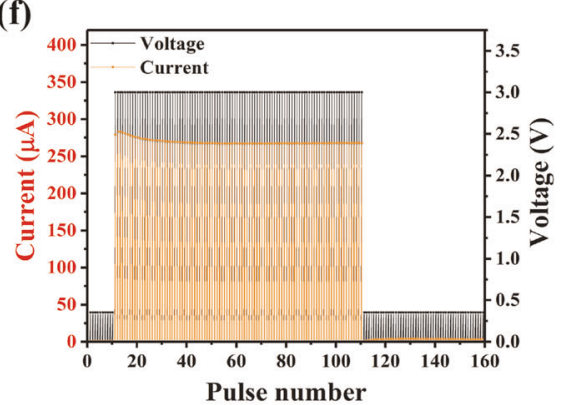

Fig. 4 Response of the post-synaptic current with various stimuli. Plasticity characteristics of the artificial synapse stimulated by 100 consecutive pulses with an amplitude of $1 \mathrm{~V}$ and pulse widths of $5 \mathrm{~ms}, 10 \mathrm{~ms}$, and $30 \mathrm{~ms}$ in a-c, respectively. Enhancement in synaptic potentiation was observed with the increasing pulse width of the stimuli, which corresponds to the increase of pain-signal with the increase of external stimuli. $\mathbf{d}$ A saturation and even inhibition in the synaptic plasticity depending on the pulse width were obtained when the stimulation voltage increased to $3 \mathrm{~V}$. 
(a)

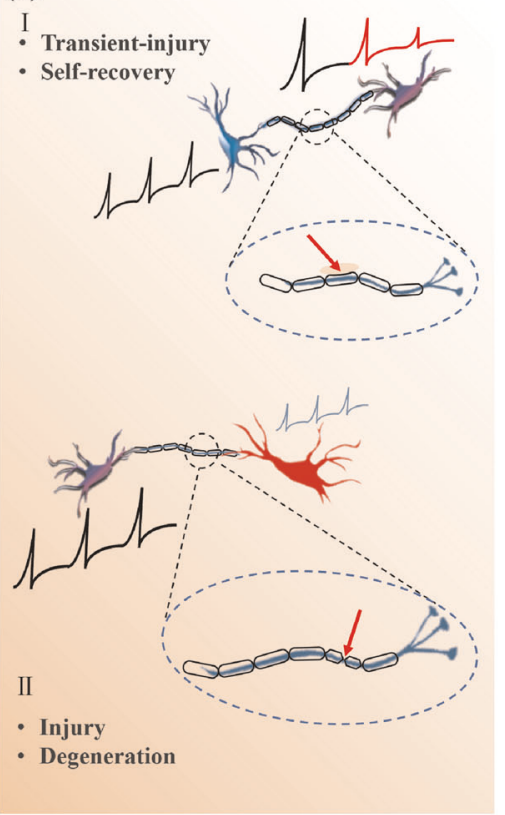

(b)
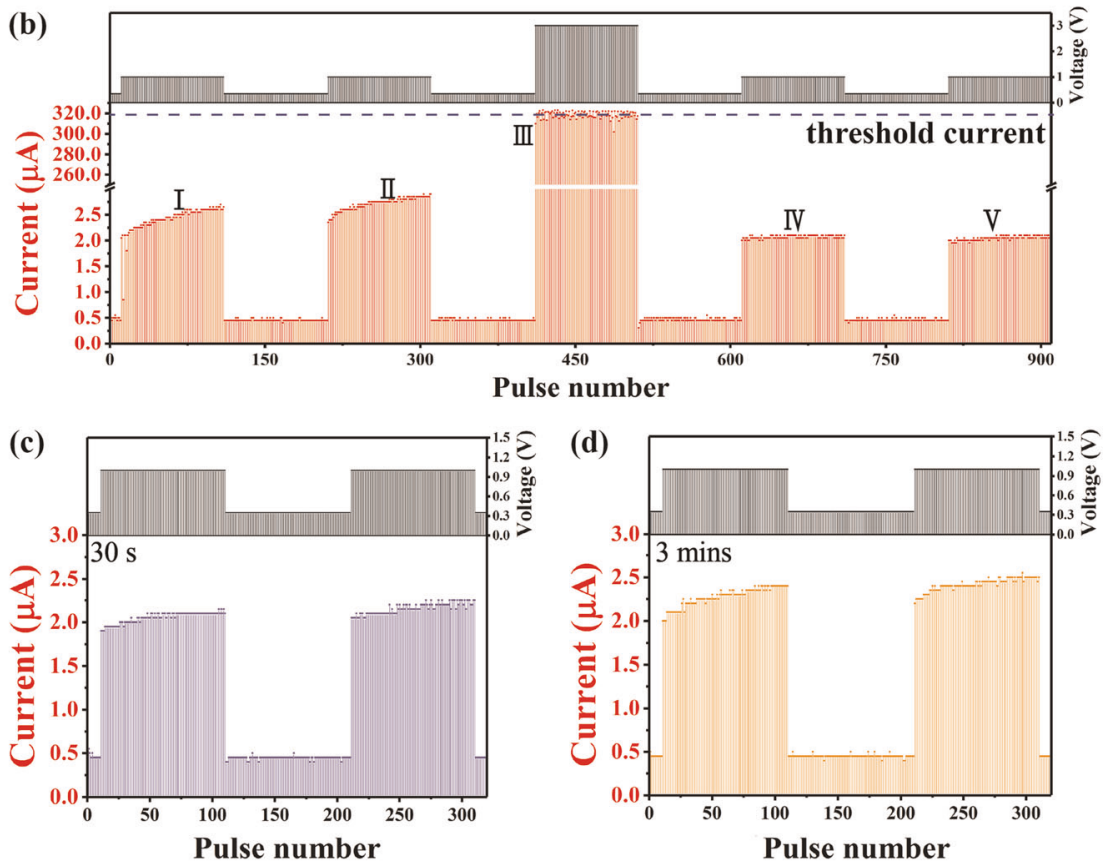

Fig. 5 Emulation of the nerve injuries by mild stimuli. a The schematic diagram for the nerve injuries caused by mild and severe stimuli. $\mathbf{b}$ Response of the post-synaptic current to mild stimuli. The dash line shows the threshold current for the functional loss of the nerve system. The self-recovery process for the nerve injuries in c $30 \mathrm{~s}$ and d $3 \mathrm{~min}$. Fully recovery of the synaptic behavior can be achieved in 3 min.

dynamics of the device. Forward and reverse IV characteristics plotted on the log scale are presented in Fig. 2c, d, respectively, to uncover the ionic behaviors from the investigation of the conduction mechanism. A current change from the lowexponent space charge limited current (SCLC) to the highexponent SCLC is observed in the forward IV curve with increasing voltage, as indicated in regions I, II, and III of Fig. 2c. The behavior is controlled by the fulfillment of the trapping sites by the carriers and diminishes the existing trap concentration gradually in order of the energy levels, which leads to an increase in the conductance. However, a decrease in conductance is achieved as the voltage further increases, as shown in region IV of Fig. 2c. This result is ascribed to the intercalation of $\mathrm{Li}^{+}$in the s-SWCNTs; in turn, the motion of $\mathrm{Li}^{+}$along the radial direction is limited due to radial confinement, leading to a lower mobility ${ }^{32}$. Regarding the reverse trace in Fig. $2 \mathrm{~d}$, a further decrease in the conductance in the high-voltage region (region IV is observed due to the continuous intercalation of $\mathrm{Li}^{+}$in the s-SWCNTs. These behaviors result in a clockwise hysteresis in this region. With the decrease in voltage (regions I, II, and III), a more conductive channel than that of forward sweeping is obtained, benefiting from the unreleased carriers in the trapping sites. As a consequence, an anticlockwise hysteretic loop is generated.

These two processes can also be supported by investigating the IV behaviors with various measurement temperatures and humidities, as shown in the Supplementary Figs 7 and 8). Except for the increase in current described by the Vogel-Tamman-Fulcher (VTF) equation $^{33}$, a clockwise hysteresis in the IV curves that gradually diminishes with increasing temperature is observed (Supplementary Fig. 7). This phenomenon is due to the fact that the structures of $\mathrm{Li}^{+}$trapped inside carbon nanotubes through topological defects in the sidewall are dependent on the temperature, and an ordered structure exists, especially at low temperatures. In contrast, the clockwise hysteresis gradually dominates the IV characteristics as the humidity decreases (Supplementary Fig. 7). This is due to the decrease in proton concentration with decreasing ambient humidity, which leads to $\mathrm{Li}^{+}$being the main carrier in the device.
Consequently, a smaller current resulted from the reduced carrier concentration, and a gradual domination of the clockwise hysteresis caused by the intercalation of $\mathrm{Li}^{+}$in the s-SWCNTs was observed. In addition, if the device was dried at $100{ }^{\circ} \mathrm{C}$ for $24 \mathrm{~h}$, it exhibited similar behavior to that measured at low humidity. That is, a clockwise hysteresis independent of the sweeping voltage is observed if the sample is fully dried as shown in Supplementary Fig. 9, which also proves that the clockwise hysteretic loop is a result of the $\mathrm{Li}^{+}$in the channel. Furthermore, the conduction mechanism of the devices measured at high temperature $\left(85^{\circ} \mathrm{C}\right)$ and under dry conditions in Supplementary Fig. 10 is also evidences for the validity of the above analysis. Therefore, it is concluded that the SCLC transition caused by the charge trapping/de-trapping process and the intercalation of $\mathrm{Li}^{+}$in the s-SWCNTs contribute to the memristive behaviors of artificial synapses. The protons and $\mathrm{Li}^{+}$ in the artificial synapse play roles resembling those of $\mathrm{Na}^{+}$and $\mathrm{K}^{+}$ in biological systems.

Based on the above discussion, a schematic diagram of the working mechanism of the artificial synapse is proposed in Fig. 3. The hopping of carriers, including protons and $\mathrm{Li}^{+}$, through functional groups and their trapping in the trapping-sites through topological defects in the sidewall occur in the low-voltage region $(0-1.4 \mathrm{~V})$, as illustrated in Fig. 3a. The presence of the functional groups and defects in the s-SWCNTs was confirmed by the XPS spectra in Fig. 1d and the Raman spectrum in Fig. 2b. With the increase in voltage, the intercalation of $\mathrm{Li}^{+}$in the s-SWCNTs occurs, as shown in Fig. 3b, which leads to a reduction in carrier concentration and mobility. Therefore, a decrease in the slope of the IV characteristic was observed.

With the understanding of the working mechanism, a possible artificial synapse, which can imitate the transmission of pain signals, is demonstrated in Fig. 4. Similar to the post-synaptic current dependent on the action potential in a biological system, the post-synaptic current of the artificial synapse can also be modulated by the stimuli of applied voltage pulses. First, the plasticity characteristics of an artificial synapse stimulated by 100 consecutive pulses with an amplitude of $1 \mathrm{~V}$ and pulse widths of 
(a)
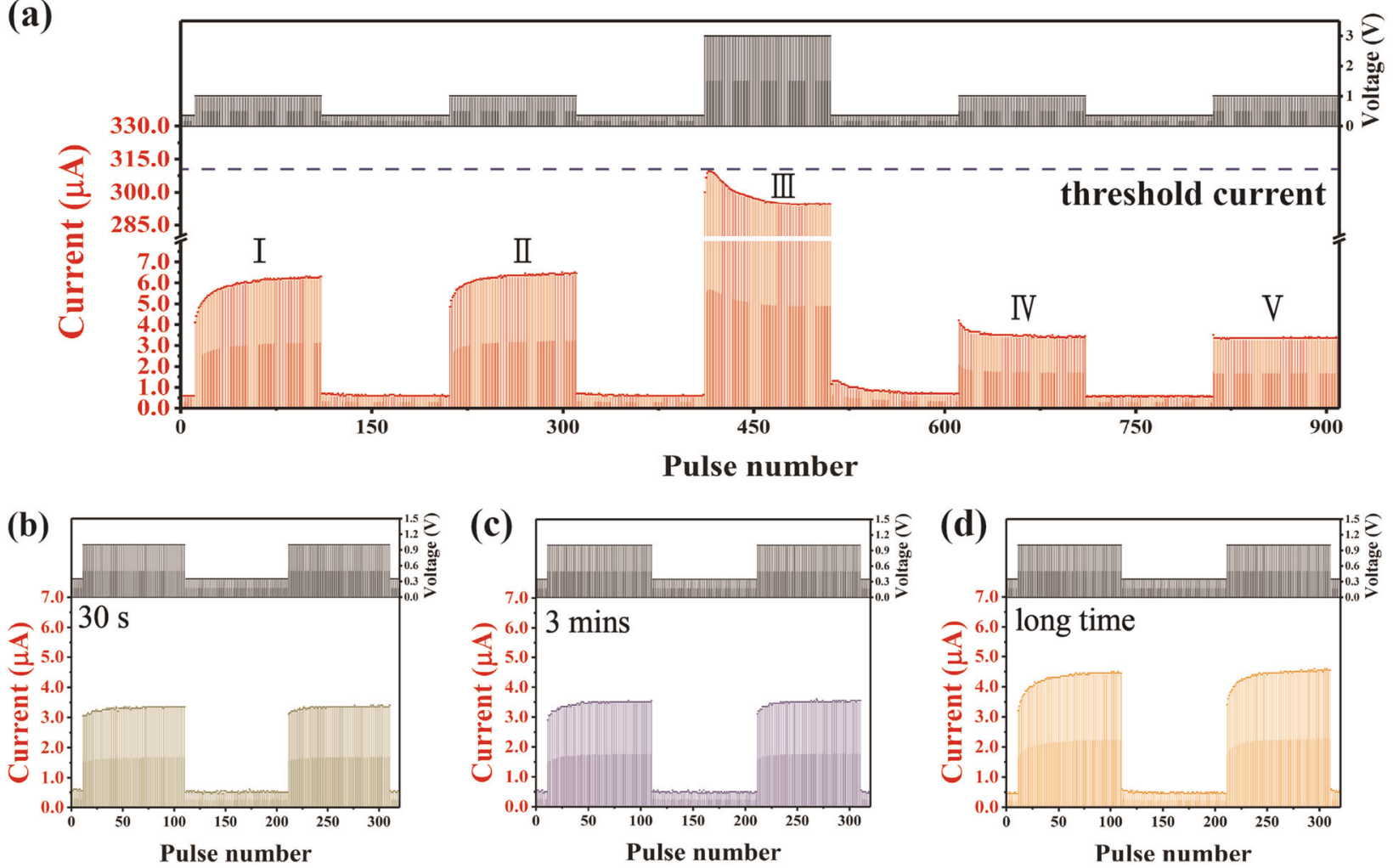

Fig. 6 Emulation of the nerve injuries by mild and severe stimuli. a Response of the post-synaptic current to severe stimuli. The dash line shows the threshold current for the functional loss of the nerve system. $\mathbf{b}$ - $\mathbf{d}$ The complete functional loss occurs and complete recovery cannot be achieved for a long time.

$5 \mathrm{~ms}, 10 \mathrm{~ms}$, and $30 \mathrm{~ms}$ were investigated and are presented in Fig. $4 a-c$, respectively. With the increase in pulse width, there was an enhancement in synaptic potentiation, which corresponded to the increase in the pain signal with the increase in external stimuli. However, when the voltage increased to $3 \mathrm{~V}$, interesting behaviors were observed. The post-synaptic current increased dramatically during the first several stimuli of $3 \mathrm{~V}$ over $10 \mathrm{~ms}$. Then, it saturated gradually, and a maximum current was obtained regardless of the member of pulses, as shown in Fig. 4a. The behavior is related to neuroprotection in cases of possible neuropathic injury of the nervous system due to the stimuli. When the pulse width increased to $30 \mathrm{~ms}$, a small decrease following the maximum post-synaptic current was observed, as shown in Fig. 4b. The decrease in current became more obvious when the pulse width was further increased to $50 \mathrm{~ms}$. In addition, fewer pulses were required to achieve the maximum current as shown in Fig. 4c. The accompanied injuries to the nervous system usually occurred in these cases. Therefore, the presented potentiation and selflimitation in the current show the potential for the emulation of pain perception, neuroprotection, and injury to the nervous system.

According to the grading systems developed by Seddon and Sunderland, nerve injuries can be divided into three categories: neurapraxia, axonotmesis, and neurotmesis based on the microscopic changes and patient symptomatology ${ }^{34,35}$. Neurapraxia, the mildest injury as a result of a transient conduction block at the site of injury, does not involve loss of nerve continuity and causes functional loss. The injury can be self-recovered in a short time. However, axonotmesis and neurotmesis usually refer to a severe or complete interruption of the nerve axon or disconnection of a nerve, which are caused by the occurrence of axon and myelin degeneration distal to the point of injury or complete denervation.
Functional loss occurs and surgical intervention is required for recovery. A schematic diagram for mild and severe nerve injuries is presented in Fig. 5a. The artificial synapse developed in this study was employed to mimic and demonstrate the responses of neural signals to pain sensation and possible nerve injuries discussed above, which is the key component for the construction of a bio-inspired perception system.

From the results in Fig. 4, it is clear that nerve injuries can be induced only for stimuli with a large voltage, $3 \mathrm{~V}$ in this study. If the pulse voltage is $1 \mathrm{~V}$, a smooth increase in the post-synaptic current is obtained, which indicates a potentiation of the artificial synapse. Therefore, in this demonstration, stimuli with a voltage of $3 \mathrm{~V}$ are used to generate different types of nerve injuries by varying the pulse width. Successive pulses with a voltage of $1 \mathrm{~V}$ are employed to induce synaptic potentiation without causing injury to the nervous system, and a voltage of $0.35 \mathrm{~V}$ is selected to check the post-synaptic current when no stimuli are applied (a discussion of the selection of $0.35 \mathrm{~V}$ as the voltage is presented in Supplementary Fig. 11). Figure 5b-d show the transient-injury of the artificial nerve stimulated by a series of mild pulses of $3 \mathrm{~V}$ over $10 \mathrm{~ms}$ and its self-recovery process. Initially, repetitive synaptic potentiation shows the proper function of the artificial synapse without any injury, as indicated by I and II in Fig. 5b. A saturation of the neural signal is observed for the stimuli of $3 \mathrm{~V}$ over $10 \mathrm{~ms}$ (indicated by III), which leads to the abnormal behaviors in the potentiation of the post-synaptic current (IV and V). Fortunately, the transient injury can be self-recovered in a short time, $<3 \mathrm{~min}$ in this study. These processes resemble neurapraxia in biological systems and can be used to mimic cases of paralysis or injury caused by the mildest stimuli.

However, complete functional loss occurs if the artificial synapse suffers from an acute painful stimulus. Additionally, the 
performance cannot be recovered to its initial state regardless of the recovery time, which resembles axonotmesis and neurotmesis in biological synapses. For the artificial synapse studied in this research, the threshold current for the functional loss of the nerve system is about $315 \mu \mathrm{A}$ as indicated in Figs $5 \mathrm{~b}$ and $6 \mathrm{a}$. Figure 6 shows the emulation of this process by increasing the pulse width of the stimuli to $30 \mathrm{~ms}$. Figure $6(\mathrm{a}-\mathrm{I})$ and $(\mathrm{a}-\mathrm{II})$ show the synaptic potentiation stimulated with pulses of $1 \mathrm{~V}$ over $30 \mathrm{~ms}$. However, an inhibition rather than potentiation in the post-synaptic current occurs as the voltage increases to $3 \mathrm{~V}$ over $30 \mathrm{~ms}$ and corresponding current reaches the threshold level, as shown in Fig. 6 (a-III), which is caused by the intercalation of $\mathrm{Li}^{+}$in $\mathrm{s}$ SWCNTs at relatively high voltage, as discussed in Fig. 4. The functional loss lasts for several seconds (Fig. 6(a-IV) and (a-V)), and complete recovery cannot be achieved for a long time, as shown in Fig. $6 b-d$, which is similar to denervation due to axon and myelin degeneration in biological systems.

\section{DISCUSSIONS}

In conclusion, a bio-inspired double-layer artificial synapse was fabricated with s-SWCNTs and PEO: $\mathrm{LiClO}_{4}$. The device exhibits synaptic potentiation in the low-voltage region and inhibition in the high-voltage region towards the emulation of pain perception and nerve injuries of living creatures. Physical and electrical characterizations were carried out to provide evidence and possible explanations for the working mechanism of the artificial synapses. The results show that the underlying mechanism is based on the hopping of carriers through functional groups and the intercalation of $\mathrm{Li}^{+}$in s-SWCNTs. Based on the fabricated artificial synapses, neurapraxia, axonotmesis, and neurotmesis caused by different nerve injuries were emulated. Overall, the artificial synapse offers a new avenue for the construction of a bioinspired perception system, which provides a solution to the intelligent human-machine interface.

\section{METHODS}

Fabrication of the flexible memristor-based artificial synapses Diluted polyimide (PI) solution was spin-coated on the cleaned glass and cured at $300^{\circ} \mathrm{C}$ for $1 \mathrm{~h}$. Then, the electrodes $(\mathrm{Ti} / \mathrm{Au}$ ) were deposited on the PI substrates by sputtering and patterned using photolithograph and liftoff method. After that, $90 \%$ s-SWCNTs (Nanolntegris) dispersed in dimethyl formamide (DMF) was drop-casted on the sample at $50^{\circ} \mathrm{C}$ followed by the bake at $160^{\circ} \mathrm{C}$ for $1 \mathrm{~h}$ and the AFM images in Supplementary Fig. 1a-C show the carbon nanotube network deposited by various methods. The active regions were defined using photolithograph and $\mathrm{O}_{2}$ plasma etching and IV characteristics before and after plasma etching are illustrated in Supplementary Fig. 1d. Afterward, the PEO: $\mathrm{LiClO}_{4}(10: 1)$ solution $(5 \mu \mathrm{L})$ was dropped on the top of active regions and baked $80^{\circ} \mathrm{C}$ for $30 \mathrm{~min}$. Finally, PI membrane was peeled-off from the glass. The schematic diagrams for the fabrication process are demonstrated in Supplementary Fig. 2 of the supplement materials.

\section{Device characterizations}

The surface morphology of s-SWCNTs were investigated by AFM (Burker Dimension). FTIR spectra were collected via a Thermo Nicolet iN 10 spectrometer with the laser working at $77 \mathrm{~K}$ in a liquid nitrogen environment. XPS analysis was carried out using Thermo Scientific EXCALAB $250 \mathrm{XI}$. All the electrical measurements were performed at $25^{\circ} \mathrm{C}$ and $40 \%$ humidity using an Agilent B1500A semiconductor parameter analyzer.

\section{DATA AVAILABILITY}

The datasets generated during and/or analyzed during the current study are available from the corresponding author on reasonable request.
Received: 11 October 2019; Accepted: 13 February 2020; Published online: 04 March 2020

\section{REFERENCES}

1. Li, X. Y. \& Toyoda, H. Role of leak potassium channels in pain signaling. Brain Res. Bull. 119, 73-79 (2015).

2. Galluzzi, K. E. Managing neuropathic pain. J. Am. Ost. Assoc. 107, ES39-ES48 (2007).

3. Waxman, S. G., Dib-Hajj, S., Cummins, T. \& Black, J. Sodium channels and pain. Proc. Natl Acad. Sci. 96, 7635-7639 (1999).

4. Bang, H., Kim, Y. \& Kim, D. TREK-2, a new member of the mechanosensitive tandem-pore $\mathrm{K}^{+}$channel family. J. Biol. Chem. 275, 17412-17419 (2000).

5. Sandoz, G., Douguet, D., Chatelain, F., Lazdunski, M. \& Lesage, F. Extracellular acidification exerts opposite actions on TREK1 and TREK2 potassium channels via a single conserved histidine residue. Proc. Natl Acad. Sci. 106, 14628-14633 (2009).

6. Julius, D. \& Basbaum, A. I. Molecular mechanisms of nociception. Nature 413, 203 (2001).

7. Eglen, R. M., Hunter, J. C. \& Dray, A. lons in the fire: recent ion-channel research and approaches to pain therapy. Trends Pharmacol. Sci. 20, 337-342 (1999).

8. Wood, J. N. et al. Ion channel activities implicated in pathological pain. Novartis Found. Symp. 1999, 32-40 (2004).

9. Eijkelkamp, N. et al. Neurological perspectives on voltage-gated sodium channels. Brain 135, 2585-2612 (2012).

10. Mathie, A. \& Veale, E. L. Two-pore domain potassium channels: potential therapeutic targets for the treatment of pain. Pflug. Arch. Eur. J. Phy. 467, 931-943 (2015).

11. Jiang, R., Ma, P., Han, Z. \& Du, X. Habituation/Fatigue behavior of a synapse memristor based on IGZO-HfO 2 thin film. Sci. Rep. 7, 9354 (2017).

12. Kim, S. G., Han, J. S., Kim, H., Kim, S. Y. \& Jang, H. W. Recent advances in memristive materials for artificial synapses. Adv. Mater. Technol. 3, 1800457 (2018).

13. Wang, Z. et al. Memristors with diffusive dynamics as synaptic emulators for neuromorphic computing. Nat. Mater. 16, 101 (2016).

14. Diorio, C., Hasler, P., Minch, B. A. \& Mead, C. A single-transistor silicon synapse. IEEE Trans. Electr. Dev. 43, 1972-1980 (1996).

15. Xie, D. et al. Coplanar multigate $\mathrm{MoS}_{2}$ electric-double-layer transistors for neuromorphic visual recognition. ACS Appl. Mater. Inter. 10, 25943-25948.

16. Bagdzevicius, S., Maas, K., Boudard, M. \& Burriel, M. Interface-type resistive switching in perovskite materials. J. Electroceram. 39, 157-184 (2017).

17. Yang, J. J. et al. The mechanism of electroforming of metal oxide memristive switches. Nanotechnology 20, 215201 (2009).

18. Sawa, A. Resistive switching in transition metal oxides. Mater. Today 11, 28-36 (2008).

19. van De Burgt, Y., Melianas, A., Keene, S. T., Malliaras, G. \& Salleo, A. Organic electronics for neuromorphic computing. Nat. Electron. 1, 386 (2018).

20. Huh, W. et al. Synaptic barristor based on phase-engineered 2D heterostructures. Adv. Mater. 30, 1801447 (2018).

21. Qiao, N. et al. A reconfigurable on-line learning spiking neuromorphic processor comprising 256 neurons and 128K synapses. Front. Neurosci. 9, 141 (2015).

22. Furber, S. B., Galluppi, F., Temple, S. \& Plana, L. A. The spinnaker project. Proc. IEEE 102, 652-665 (2014)

23. Osborn, L., Kaliki, R. R., Soares, A. B. \& Thakor, N. V. Neuromimetic event-based detection for closed-loop tactile feedback control of upper limb prostheses. IEEE Trans. Haptics 9, 196-206 (2016).

24. Beer, R. D., Quinn, R. D., Chiel, H. J. \& Ritzmann, R. E. Biologically Inspired. Commun. ACM 40, 31 (1997).

25. Goldschmidt, D., Wörgötter, F. \& Manoonpong, P. Biologically-inspired adaptive obstacle negotiation behavior of hexapod robots. Front. Neurosci. 8, 3 (2014).

26. Zaghloul, K. A. \& Boahen, K. A silicon retina that reproduces signals in the optic nerve. J. Neural Eng. 3, 257 (2006).

27. Tee, B. C. K. et al. A skin-inspired organic digital mechanoreceptor. Science 350, 313-316 (2015).

28. Qiu, S. et al. Solution-processing of high-purity semiconducting single-walled carbon nanotubes for electronics devices. Adv. Mater. 31, 1800750 (2019).

29. Goldstein, S. A., Bockenhauer, D., O'Kelly, I. \& Zilberberg, N. Potassium leak channels and the KCNK family of two-P-domain subunits. Nat. Rev. Neurosci. 2, 175 (2001).

30. Qiu, H. et al. Hopping transport through defect-induced localized states in molybdenum disulphide. Nat. Commun. 4, 2642 (2013).

31. Shang, D. S. et al. Effect of carrier trapping on the hysteretic current-voltage characteristics in $\mathrm{Ag} / \mathrm{La}_{0} \mathrm{Ca}_{03} \mathrm{MnO}_{3} / \mathrm{Pt}$ heterostructures. Phys. Rev. B 73, 245427 (2006).

32. Zhao, M. et al. Curvature-induced condensation of lithium confined inside singlewalled carbon nanotubes: first-principles calculations. Phys. Lett. A 340, 434-439 (2005). 
33. Zhou, D., Mei, X. \& Ouyang, J. lonic conductivity enhancement of polyethylene oxide- $\mathrm{LiClO}_{4}$ electrolyte by adding functionalized multi-walled carbon nanotubes. J. Phys. Chem. C. 115, 16688-16694 (2011).

34. Seddon, H. Three types of nerve injury. Brain 66, 237-288 (1943).

35. Burnett, M. G. \& Zager, E. L. Pathophysiology of peripheral nerve injury: a brief review. Neurosurg. Focus 16, 1-7 (2004).

\section{ACKNOWLEDGEMENTS}

We acknowledge the funding support from the National Key R\&D Program of China (No. 2018YFB1304700), the National Natural Science Foundation of China (61574163), the Science Foundation for Distinguished Young Scholars of Jiangsu Province, China (BK20170008, BK20160011), the National Natural Science Foundation of China (61801473), and the NANO-X Workstation scientifically supported this research.

\section{AUTHOR CONTRIBUTIONS}

Q.L., F.S., and T.Z. designed the project and experiments. Q.L, L.L., and L.L prepared the manuscript. M.H. and Z.W. analyzed and discussed the data. All authors reviewed the manuscript.

\section{COMPETING INTERESTS}

The authors declare no competing interests.

\section{ADDITIONAL INFORMATION}

Supplementary information is available for this paper at https://doi.org/10.1038/ s41528-020-0066-0.

Correspondence and requests for materials should be addressed to T.Z.

Reprints and permission information is available at http://www.nature.com/ reprints

Publisher's note Springer Nature remains neutral with regard to jurisdictional claims in published maps and institutional affiliations.

cc) (i)

Open Access This article is licensed under a Creative Commons Attribution 4.0 International License, which permits use, sharing, adaptation, distribution and reproduction in any medium or format, as long as you give appropriate credit to the original author(s) and the source, provide a link to the Creative Commons license, and indicate if changes were made. The images or other third party material in this article are included in the article's Creative Commons license, unless indicated otherwise in a credit line to the material. If material is not included in the article's Creative Commons license and your intended use is not permitted by statutory regulation or exceeds the permitted use, you will need to obtain permission directly from the copyright holder. To view a copy of this license, visit http://creativecommons. org/licenses/by/4.0/.

(c) The Author(s) 2020 